\title{
Embedding holomorphic discs through discrete sets
}

\author{
Franc Forstneric ${ }^{1, \star}$, Josip Globevnik ${ }^{2, \star \star}$, Berit Stensønes ${ }^{3}$ \\ ${ }^{1}$ Department of Mathematics, University of Wisconsin, Madison, WI 53706, USA \\ ${ }^{2}$ Institute of Mathematics, Physics and Mechanics, Jadranska 19, SI-61000 Ljubljana, Slovenia \\ ${ }^{3}$ Department of Mathematics, University of Michigan, Ann Arbor, MI 48109, USA
}

Received: 31 May 1995

Mathematics Subject Classification (1991): 32H02, 32H35, 32M05

\section{Introduction and the main result}

We denote by $D$ the open unit disc in $C: D=\{\zeta \in C:|\zeta|<1\}$. A domain $\Omega \subset C^{n}$ is said to be Runge in $C^{n}$ if every holomorphic function in $\Omega$ can be approximated, uniformly on compacts in $\Omega$, by restrictions to $\Omega$ of holomorphic polynomials on $C^{n}$. We refer the reader to Hörmander [12] for general results concerning pseudoconvex Runge domains. Our main result is

Theorem. Given a connected pseudoconvex Runge domain $\Omega \subset C^{n}(n \geqq 2)$ and a discrete subset $Z=\left\{z_{j}: j \in Z_{+}\right\} \subset \Omega$, there exists a proper holomorphic embedding $f: D \rightarrow \Omega$ of the dise into $\Omega$ whose image $f(D)$ contains $Z$.

The most interesting case is $n=2$. For $n \geqq 3$ this was proved by a different method in [16] for $\Omega=C^{n}$ and in [9] for all convex domains $\Omega \subset C^{n}$. In the case when $\Omega=C^{n}(n \geqq 3)$ one can in addition prescribe a discrete set $\left\{\zeta_{j}\right\} \subset C$ and require that $f\left(\zeta_{j}\right)=z_{j}$ for all $j$ [16].

For $n=2$ the methods of [9] and [16] only give proper holomorphic immersions of the disc through a given discrete set in $\Omega$. The main problem of course is that, in dimension two, one cannot remove self-intersections of complex curves by small deformations.

We do not know whether our Theorem holds for non-Runge pseudoconvex domains in $C^{n}$; our methods do not seem to extend to this case. In this direction it was proved in [6] that for every finite subset $Z$ in an arbitrary connected pseudoconvex domain $\Omega \subset C^{n}(n>1)$ there exist proper holomorphic mappings $f: D \rightarrow \Omega$ of the disc into $\Omega$ such that $Z \subset f(D)$. It is likely that a refinement of the construction in [6] gives proper holomorphic immersions $f: D \rightarrow \Omega$ whose image contains a given discrete subset of $\Omega$. An example

* Supported in part by an NSF grant and by a grant from the Ministry of Science of the Republic of Slovenia,

$\star \star$ Supported in part by a grant from the Ministry of Science of the Republic of Slovenia 
in [6] shows that the Theorem does not extend to arbitrary non-pseudoconvex domains.

In this paper we develop an essentially new approach which works in every dimension $n \geqq 2$. Our methods were inspired in part by the earlier work of two of the authors [10]. The main idea is to construct a sequence of proper holomorphic embeddings $f_{k}$ : $\boldsymbol{C} \rightarrow \boldsymbol{C}^{n}$ such that the points $z_{0}, \ldots, z_{k}$ are contained in the same connected component of $f_{k}(C) \cap \Omega$. The next map $f_{k+1}$ is of the form $f_{k+1}=\Phi_{k} \circ f_{k}$, where $\Phi_{k}$ is a suitably chosen holomorphic automorphism of $C^{n}$ which is very close to the identity on a prescribed polynomially convex set $K_{k} \subset \subset \Omega$, it fixes the points $z_{0}, \ldots, z_{k}$, and it moves the image variety outside $K_{k}$ so that $f_{k+1}(C)$ contains the next point $z_{k+1} \in Z$ as well.

A similar but technically simpler construction was used in [7] to construct proper holomorphic embeddings $f: C \rightarrow C^{2}$ of the complex line into $C^{2}$ whose image $f(C)$ contains a given discrete set $Z \subset C^{2}$. The additional difficulty in the present paper is to keep the points $z_{0}, \ldots, z_{k}$ in the same connected component of $f_{k}(C) \cap \Omega$ at each step. To achieve this we need the following tool which, we hope, will be of independent interest. It is an extension of some earlier results of Rosay and one of the authors [8] (and whose proof is based in part on the ground breaking work by Andérsen [3] and Andersén and Lempert [4]).

Proposition (Combing hair by holomorphic automorphisms). Let $K \subset \subset C^{n}$ $(n \geqq 2)$ be a compact polynomially convex set and let $C \subset C^{n}$ be a smooth embedded arc of class $\mathcal{C}^{r}, r \geqq 3$, (a diffeomorphic image of $[0,1] \subset R$ ) which is attached to $K$ in a single point of $K$. Given a homeomorphism $F: K \cup$ $C \rightarrow K \cup C^{\prime} \subset C^{n}$ such that $F$ is the identity on $(K \cup C) \cap U$ for some open neighborhood $U \subset C^{n}$ of $K$, there exists for each $\varepsilon>0$ a global holomorphic automorphism $\Phi$ of $C^{n}$ satisfying $|\Phi(z)-F(z)|<\varepsilon$ for $z \in K \cup C$. Moreover, for each pair of finite subsets $A \subset C, B \subset C^{n} \backslash C$, there is a $\Phi$ as above such that $\left.\Phi\right|_{A}=\left.F\right|_{A}$ and $\Phi$ fixes $B$ pointwise.

Remarks. 1. The same result holds for any finite number of disjoint hair attached to $K$. In other words, one can comb hair on a polynomially convex head in $C^{n}(n \geqq 2)$ approximately by global holomorphic automorphisms of $C^{n}$.

2. Similar results for arcs without the presence of the polynomially convex set $K$ have been proved by Rosay [14] and Forstneric [5]. Our proof of the proposition can easily be refined to show that, if $F$ as above is a $\mathcal{C}^{r}$ diffeomorphism on $C$ and $r \geqq 3$, then an approximating sequence $\Phi_{j} \in$ Aut $C^{n}$ can be chosen such that $\left.\Phi_{j}\right|_{C}$ converges to $\left.F\right|_{C}$ in $C^{r-3}(C)$. This result is not sharp, the loss of derivatives being due to the fact that we are using Hörmander's $L^{2}$-method for solving a $\bar{\partial}$ equation in certain thin tubes. By using a more precise method such as the one in [5] or in [13] one expects no loss of derivatives as soon as $r \geqq 2$. 
The proposition is proved in Sect. 2. Granted the proposition we shall now prove the Theorem. We will need the following Lemma (which is not original).

Lemma. If $\Omega \subset C^{n}$ is a pseudoconvex Runge domain and if $f: C \rightarrow C^{n}$ is a proper holomorphic embedding, then each connected component of $f(C) \cap \Omega$ is simply connected and hence biholomorphic either to the disc or to $C$.

Proof. Set $A=f(C)$. Let $A$ be a connected component of $A \cap \Omega$ and let $U=$ $\{\zeta \in C: f(\zeta) \in A\}$. We must show that $U$ is simply connected. If not, choose a point $\zeta_{0}$ in a bounded component of $C \backslash U$. Define a holomorphic function $F: A \rightarrow C$ by $F(f(\zeta))=1 /\left(\zeta-\zeta_{0}\right)$. By Cartan's theorem A [12] $F$ extends to a holomorphic function in $\Omega$. Since $\Omega$ is Runge in $C^{n}, F$ can be approximated by holomorphic polynomials $P_{j}$. Then $P_{j} \circ f$ is a sequence of entire functions on $C$ which converges to the function $\zeta \rightarrow 1 /\left(\zeta-\zeta_{0}\right)$ uniformly on compacts in $U$. Clearly this is a contradiction.

Proof of the Theorem. We shall first consider the case $\Omega \neq C^{n}$. Choose a smooth plurisubharmonic exhaustion function $\rho: \Omega \rightarrow R$ such that $\left.\rho\right|_{Z}$ is one to one. We shall repeatedly use the fact that for each $R \in R$ the set

$$
\Omega(R)=\{z \in \Omega: \rho(z) \leqq R\}
$$

is polynomially convex in $C^{n}$ (see Hörmander [12]). By reordering the points in $Z$ we may assume $\rho\left(z_{j}\right)<\rho\left(z_{j+1}\right)$ for each $j \in Z_{+}$. Choose numbers $R_{j}$ $(j=0,1,2, \ldots)$ such that

$$
\rho\left(z_{j}\right)<R_{j}<\rho\left(z_{j+1}\right)
$$

and set $K_{j}=\Omega\left(R_{j}\right)$. Then $\left\{K_{j}\right\}$ is an increasing sequence of compact polynomially convex sets such that $\bigcup_{0 \leq j<\infty} K_{j}=\Omega$. We also have $K_{j} \cap Z=$ $\left\{z_{0}, z_{1}, \ldots, z_{j}\right\}$ and $\left(K_{j+1} \backslash K_{j}\right) \cap Z=\left\{z_{j+1}\right\}$.

The initial step. Fix a point $a \in C^{n} \backslash \Omega$. Let $\zeta_{0}=0 \in C$. Choose a proper holomorphic embedding $f_{0}: C \rightarrow C^{n}$ such that $f(0)=z_{0}, f(1)=a$, and $\Lambda_{0}=$ $f_{0}(C)$ does not contain the point $z_{1}$. Set $L_{-1}=\varnothing, \Delta_{-1}=\varnothing$, and $V_{-1}=\varnothing$.

The inductive step. Let $k \in \boldsymbol{Z}_{+}$. Suppose we already have a proper holomorphic embedding $f_{k}: C \rightarrow C^{n}$ with image $A_{k}=f_{k}(C)$, a set of points $\left\{\zeta_{0}, \zeta_{1}, \ldots, \zeta_{k}\right\} \subset C \backslash\{1\}$, a number $M_{k-1}$, and a smoothly bounded, simply connected domain $\Delta_{k-1} \subset \subset C \backslash\{1\}$ such that

(i) $f_{k}\left(\zeta_{j}\right)=z_{j}$ for $0 \leqq j \leqq k$,

(ii) $f_{k}(1)=a$,

(iii) $z_{k+1} \notin \Lambda_{k}$, and

(iv) the set $\left\{\zeta_{0}, \zeta_{1}, \ldots, \zeta_{k}\right\} \cup \overline{\Delta_{k-1}}$ is contained in one connected component $U_{k}^{0}$ of the set

$$
U_{k}=\left\{\zeta \in C: f_{k}(\zeta) \in \Omega\right\}
$$


Note that $\rho \circ f_{k}$ is an exhaustion function on $U_{k}$. Choose a number

$$
M_{k} \geqq \max \left(R_{k}, M_{k-1}\right)+1
$$

which is a regular value of $\left.\rho \circ f_{k}\right|_{U_{k}}$ and such that $\left\{\zeta_{0}, \zeta_{1}, \ldots, \zeta_{k}\right\} \cup \overline{\Delta_{k-1}}$ is contained in one connected component $\Delta_{k}$ of the set

$$
V_{k}=\left\{\zeta \in U_{k}: \rho \circ f_{k}(\zeta)<M_{k}\right\} \subset \subset U_{k} .
$$

$V_{k}$ consists of finitely many smoothly bounded components $\Delta_{k}=\Delta_{k}^{0}, \Delta_{k}^{1}, \ldots$, $\Delta_{k}^{j k}$ which are simply connected and have disjoint closures.

Set

$$
L_{k}=K_{k} \cup\left(\Lambda_{k} \cap \Omega\left(M_{k}\right)\right)=K_{k} \cup \overline{f_{k}\left(V_{k}\right)} .
$$

We claim that $L_{k}$ is polynomially convex. Suppose that a point $z \in C^{n} \backslash L_{k}$ belongs to the polynomial hull $\hat{L}_{k}$. Then $z \in \Omega\left(M_{k}\right)$ since the set $\Omega\left(M_{k}\right)$ is polynomially convex and it contains $L_{k}$. Thus $z \notin \Lambda_{k} \cup K_{k}$. By Cartan's theorem A [12] there exists a holomorphic function $g$ on $C^{n}$ such that $g(z)=1$ and $g=0$ on $A_{k}$. Since $K_{k}$ is polynomially convex, there exists another holomorphic function $h$ on $C^{n}$ such that $h(z)=1$ and $\sup _{K_{k}}|h|<1$. The holomorphic function $G=g h^{N}$ for sufficiently large $N>0$ satisfies $G(z)=1$ and $\sup _{L_{k}}|G|<1$. This contradiction shows that $L_{k}=\hat{L}_{k}$ as claimed.

We now choose a smooth arc $\lambda_{k} \subset U_{k}^{0} \backslash \Delta_{k}$ which is attached to $\bar{\Delta}_{k}$ in a single point and which does not intersect any other set $\overline{\Delta_{k}^{j}}$ for $1 \leqq j \leqq j_{k}$. Denote the other endpoint of $\lambda_{k}$ by $\zeta_{k+1}$. Hence $C_{k}=f_{k}\left(\lambda_{k}\right)$ is an arc in $A_{k} \cap \Omega$ with one endpoint $f_{k}\left(\zeta_{k+1}\right)$ which is attached to $f\left(\bar{\Delta}_{k}\right) \subset L_{k}$ in the other endpoint.

By the Proposition, applied to the polynomially convex set $L_{k} \cup C_{k}$, we can find for any given $\varepsilon_{k}>0$ a holomorphic automorphism $\Phi_{k}$ of $C^{n}$ satisfying

(a) $\left|\Phi_{k}(z)-z\right|<\varepsilon_{k}$ for $z \in L_{k}$,

(b) $\Phi_{k}\left(f_{k}\left(\zeta_{k+1}\right)\right)=z_{k+1}$,

(c) $\Phi_{k}\left(z_{j}\right)=z_{j}$ for $0 \leqq j \leqq k$ and $\Phi_{k}(a)=a$,

(d) $\Phi_{k}\left(C_{k}\right) \subset \Omega$, and

(e) $\Phi_{k}\left(\Lambda_{k}\right)$ does not contain $z_{k+2}$.

Set

$$
f_{k+1}=\Phi_{k} \circ f_{k}: C \rightarrow C^{n}, \quad A_{k+1}=f_{k+1}(C) .
$$

Clearly $f_{k+1}$ is a proper holomorphic embedding of $C$ into $C^{n}$ which satisfies the properties (i)-(iv) above, with $k$ replaced by $k+1$.

Remark. It is important to observe that the point $z_{k+1}$ belongs to the same connected component of $\Lambda_{k+1} \cap \Omega$ as the points $z_{0}, \ldots, z_{k}$ since the automorphism $\Phi_{k}$ maps the arc $C_{k}$ (connecting $f_{k}\left(\zeta_{k+1}\right)$ to $f_{k}\left(\bar{\Delta}_{k}\right)$ ) into $\Omega$. If $\Omega=C^{n}$, we do not have to worry about this, and consequently one can replace our Proposition with a much simpler result to the effect that, given a polynomially convex set $K \subset \subset C^{n}$, one can move a point $p \in C^{n} \backslash K$ to a point $q \in C^{n} \backslash K$ by an automorphism of $C^{n}$ which is arbitrary close to the identity on $K$. This approach was used in [7] to construct proper holomorphic embeddings of the complex line $C$ into $C^{2}$ passing through a given discrete set $Z \subset C^{2}$. 
Completion of the proof. Set

$$
V=\bigcup_{0 \leqq k<\infty} V_{k}, \quad \Delta=\bigcup_{0 \leqq k<\infty} \Delta_{k},
$$

where $V_{k}$ is given by (2) and $\Delta_{k}=\Delta_{k}^{0}$ is the connected component of $V_{k}$ as above. Then $\left\{\zeta_{j}: j \in Z_{+}\right\} \subset \Delta \subset V \subset C \backslash\{1\}$. By property (a) of $\Phi_{k}$ we have

$$
\left|f_{k+1}-f_{k}\right|<\varepsilon_{k} \text { on } V_{k} \text {. }
$$

In each step of the construction we choose the number $\varepsilon_{k}>0$ so that the following hold:

1.) $\varepsilon_{k} \leqq \varepsilon_{k-1} / 2$ for each $k \geqq 1$,

2.) $2 \varepsilon_{k}<d\left(L_{k}, C^{n} \backslash \Omega\left(M_{k}+1\right)\right)$,

3.) $2 \varepsilon_{k}<d\left(K_{k-1}, C^{n} \backslash K_{k}\right)$.

Here $d(K, L)$ denote the Euclidean distance between the sets $K$ and $L$. Since $M_{k+1} \geqq M_{k}+1$ by (1), properties 1.) and 2.) of the sequence $\varepsilon_{k}$ insure that

$$
V_{k} \subset \subset V_{k+1}, \quad k \in Z_{+},
$$

and the sequence $f_{k}: C \rightarrow C^{n}$ converges to a limit map

$$
f=\lim _{k \rightarrow \infty} f_{k}: V \rightarrow C^{n}
$$

uniformly on compacts $\bar{V}_{k} \subset \subset$ V. On $V_{k}$ we have

$$
\left|f-f_{k}\right| \leqq \sum_{j=k}^{\infty}\left|f_{j+1}-f_{j}\right|<\sum_{j=k}^{\infty} \varepsilon_{j}<2 \varepsilon_{k} .
$$

Hence $f\left(V_{k}\right) \subset \Omega$ and therefore $f(V) \subset \Omega$.

Since $f_{k}: C \rightarrow C^{n}$ is an embedding for each $k$, we can insure by choosing $\varepsilon_{k}>0$ sufficiently small that any holomorphic map $g: C \rightarrow C^{n}$ satisfying $\mid f_{k}-$ $g \mid<2 \varepsilon_{k}$ on $V_{k}$ is an embedding on the smaller set $V_{k-1} \subset \subset V_{k}$. Thus the limit map $f: V \rightarrow \Omega$ is an injective immersion into $\Omega$.

We claim that $f: V \rightarrow D$ is also proper. Since $\left|\Phi_{k}(z)-z\right|<\varepsilon_{k}$ for $z \in K_{k}$ according to (a), the conditions 1.) and 3.) on $\varepsilon_{k}$ imply that no point from $C^{n} \backslash K_{k}$ will enter the smaller set $K_{k-1}$ after $k$-th step of the construction. Since $f_{k}\left(C \backslash V_{k}\right) \subset C^{n} \backslash K_{k}$, it follows that

$$
f\left(V \backslash V_{k}\right) \subset \Omega \backslash K_{k-1}, \quad k \in Z_{+},
$$

and hence $f$ is proper as claimed.

To summarize, we have constructed a proper holomorphic embedding $f: V \rightarrow \Omega$. Since $\Delta_{k}$ is a connected component of $V_{k}$ for each $k$, (4) implies that $\Delta$ is a connected component of $V$. Hence the restriction $f: \Delta \rightarrow \Omega$ is a proper holomorphic embedding of $\Delta$ into $\Omega$. Property (c) of $\Phi_{k}$ implies $f\left(\zeta_{k}\right)=f_{k}\left(\zeta_{k}\right)=z_{k}$ for each $k \in Z$, and hence $f(\Delta)$ contains the given discrete set $Z$. Since $\Delta$ is an increasing union of connected and simply connected domains $\Delta_{k} \subset C \backslash\{1\}, \Delta$ is itself a simply connected domain in $C \backslash\{1\}$ and 
hence biholomorphic to the unit disc $D$. Thus the map $f: \Delta \rightarrow \Omega$ satisfies our Theorem.

This proves the Theorem when $\Omega$ is a proper subdomain in $C^{n}$. In the remaining case $\Omega=C^{n}$ we can either apply the previous proof to a FatouBieberbach Runge domain $\Omega \subset C^{n}$ (which is biholomorphically equivalent to $C^{n}$ ), or else we construct the sequence $f_{k}$ as above such that $f_{k}(1)$ diverges to infinity (so $1 \notin V$ ).

\section{Combing hair by holomorphic automorphisms}

In this section we prove the Proposition stated in Sect. 1. The last requirement concerning the behavior of $\Phi$ on a finite set is a trivial addition since one can move a finite set of points for a small distance in any direction by a finite composition of shear automorphisms which are close to the identity on a chosen compact subset (see [15]). Hence it suffices to prove the first part of the proposition.

By approximation we may (and shall) assume that $F: C \rightarrow C^{\prime}$ is a $\mathcal{C}^{r}$ diffeomorphism onto another embedded $\mathcal{C}^{r}$ arc, and $F$ is the identity near $K$. Shrinking the neighborhood $U$ of $K$ if necessary we may assume that $C \cap \bar{U}=C^{\prime} \cap \bar{U}$. We extend $F$ as the identity on $\bar{U}$. Choose a one parameter family of $\mathcal{C}^{r}$ diffeomorphisms $F_{t}: \bar{U} \cup C \rightarrow \bar{U} \cup C_{t} \subset C^{n}$, smooth with respect to $0 \leqq t \leqq 1$, such that the $t$-derivative $d F_{t} / d t$ is also of class $\mathcal{C}^{r}$ and the following hold:

(i) $F_{0}$ is the identity on $\bar{U} \cup C$,

(ii) $F_{1}=F$, and

(iii) $\left.F_{t}\right|_{\bar{U}}$ is the identity for each $0 \leqq t \leqq 1$.

Let $C_{t}=F_{t}(C)$. Observe that $C_{t} \cap \bar{U}=C \cap \bar{U}$ for all $t \in[0,1]$. Let $X_{t}: U U$ $C_{t} \rightarrow C^{n}$ be the velocity vector field of $F_{t}$, defined by the equation

$$
\frac{d}{d t} F_{t}(z)=X_{t}\left(F_{t}(z)\right), \quad z \in U \cup C, 0 \leqq t \leqq 1 .
$$

Then $X_{t}$ is of class $\mathcal{C}^{r}$ in both variables $(z, t)$ and $\left.X_{t}\right|_{U}=0$ for each $t$. Thus $F_{t}(z)(z \in U \cup C)$ is the flow of the time dependent vector field $X_{t}$.

To simplify the analysis we include the parameter $t$ as an additional complex variable. Define the following subsets in $C^{n+1}$ :

$$
S=\bigcup_{0 \leqq t \leqq 1} C_{t} \times\{t\}, \quad L_{0}=K \times[0,1], \quad L=L_{0} \cup S .
$$

Since the set $K \cup C_{t} \subset C^{n}$ is polynomially convex for each $t \in[0,1]$ (Stolzenberg [17] and Alexander [2]), the sets $L_{0}$ and $L$ are polynomially convex in $C^{n+1}$.

Let $U^{\prime} \subset \subset C$ be a neighborhood of the segment $[0,1] \subset R \subset C$, and let $U_{0}=U \times U^{\prime} \subset \subset C^{n+1}$ be the corresponding neighborhood of $L_{0}$. We define a 
mapping $X: U_{0} \cup S \rightarrow C^{n}$ by

$$
X(z, t)=X_{t}(z), \quad z \in C_{t}, 0 \leqq t \leqq 1,
$$

and $\left.X\right|_{U_{0}}=0$.

Note that $S \subset C^{n+1}$ is a totally real submanifold of class $\mathcal{C}^{r}$. Since $X$ is of class, $C^{r}$ on $S$ and zero on $U_{0}, X$ extends to a map $X: C^{n+1} \rightarrow C^{n}$ of class $\mathcal{C}^{r}$, with compact support, such that

$$
\bar{\partial} X(\zeta)=o\left(d(\zeta, S)^{r-1}\right)
$$

(see Lemma 4.3 in [11]). Here, $\bar{\partial}$ is taken with respect to the variables $\zeta=$ $(z, t) \in C^{n+1}$, and $d(\zeta, S)$ denotes the Euclidean distance from $\zeta$ to $S$.

For each compact set $K \subset \boldsymbol{R}^{m}$ we denote $K(\varepsilon)=\left\{z \in \boldsymbol{R}^{m}: d(z, K)<\varepsilon\right\}$.

The Proposition follows immediatelly from the following three lemmas.

Lemma 1. (Notation as above) Assume $r \geqq 3$. There is an $\varepsilon_{0}>0$ and a continuous finction $\eta: \boldsymbol{R}_{+} \rightarrow \boldsymbol{R}_{+}, \eta(t)>0$ for $t>0, \eta(0)=0$, such that for each $0<\varepsilon \leqq \varepsilon_{0}$ there exists an entire holomorphic mapping $Y_{\varepsilon}: C^{n+1} \rightarrow C^{n}$ satisfying

$$
\left\|X-Y_{\varepsilon}\right\|_{L^{\infty}(L(\varepsilon))} \leqq \eta(\varepsilon) \varepsilon .
$$

(The function $\eta$ depends on the dimension $n$, on the set $L$, and on the vector field X.)

Recall [1] that each Lipschitz time dependent vector field $X_{t}$ on $\boldsymbol{R}^{n}$ has a local flow $\phi_{t}$ satisfying

$$
\frac{d}{d t} \phi_{t}(x)=X_{t}\left(\phi_{t}(x)\right), \quad \phi_{0}(x)=x
$$

Lemma 2. Let $X_{t}$ and $Y_{t}(0 \leqq t \leqq 1)$ be time dependent Lipschitz vector fields on $R^{n}$ with local flows $\phi_{t}$ resp. $\psi_{t}$. Assume that the flow $\phi_{t}(x)$ is defined for all $x \in K \subset \subset R^{n}$ and $0 \leqq t \leqq 1$. Set $K_{t}=\phi_{t}(K)$, and let

$$
\begin{aligned}
A(\varepsilon) & =\sup \left\{\left|X_{t}(x)-Y_{t}(x)\right|: x \in K_{t}(\varepsilon), 0 \leqq t \leqq 1\right\}, \\
B & =\sup \left\{\left|X_{t}(x)-X_{t}(y)\right|: x, y \in K_{t}(1), 0 \leqq t \leqq 1\right\} .
\end{aligned}
$$

If $A(\varepsilon) e^{B} \leqq \varepsilon \leqq 1$, then the flow $\psi_{t}(x)$ of $Y_{t}$ is defined for all $x \in K$ and $0 \leqq t \leqq 1$, and

$$
\left|\phi_{t}(x)-\psi_{t}(x)\right| \leqq A(\varepsilon) e^{B t}, \quad x \in K, 0 \leqq t \leqq 1 .
$$

In particular we have $\psi_{t}(x) \in K_{t}(\varepsilon)$ for $x \in K$ and $0 \leqq t \leqq 1$.

Lemma 3. Let $Y_{t}$ be an entire vector field on $C^{n}$ for each $0 \leqq t \leqq 1$, of class $\mathcal{C}^{1}$ in $(z, t) \in C^{n} \times[0,1]$. Let $\Omega$ be an open subset of $C^{n}$. Assume that the differential equation $d R / d t=Y_{t}(R(t))$ can be integrated for $0 \leqq t \leqq 1$ with arbitrary initial condition $R(0)=z \in \Omega$. Set $G_{t}(z)=R(t)$ as above. Then $G_{t}(0 \leqq t \leqq 1)$ is a biholomorphic map from $\Omega$ into $C^{n}$ that can be approximated, uniformly on compact sets in $\Omega$, by holomorphic automorphisms of $\mathrm{C}^{n}$. 
Lemma 3 is proved in [8] (Lemma 1.4), using results of Andersén [3] and Andersén and Lempert [4]. Although it is stated there only for time independent fields, the proof applies to time dependent entire fields as well. Lemmas 1 and 2 are proved below.

Granted these lemmas we can complete the proof of the Proposition as follows. Fix an $\varepsilon>0$ for which Lemma 1 holds. Using Lemma 1 we approximate $X$ by an entire map $Y: C^{n+1} \rightarrow C^{n}$ such that the estimate (5) is satisfied. If $\varepsilon$ is sufficiently small, Lemma 2 shows that the flow $G_{t}(z)(z \in K \cup C)$ of the time dependent holomorphic vector field $Y_{t}=Y(\cdot, t): C^{n} \rightarrow C^{n}$ exists and remains in the $\varepsilon$-neighborhood $K_{t}(\varepsilon)$ of $K_{t}=K \cup C_{t} \subset C^{n}$ for all $0 \leqq t \leqq 1$, and we have

$$
\left|F_{t}(z)-G_{t}(z)\right|<\varepsilon, \quad z \in K \cup C, 0 \leqq t \leqq 1 .
$$

Applying Lemma 3 for $t=1$ we get a $\Phi \in$ Aut $C^{n}$ such that $\left\|\Phi-G_{1}\right\|_{L^{\infty}(K \cup C)}$ $<\varepsilon$. Hence $\|\Phi-F\|_{L^{\infty}(K \cup C)}<2 \varepsilon$.

Proof of Lemma 1. This is essentially proved in the paper by Hörmander and Wermer [11] (proof of Theorem 4.1, pp. 15-16), except that the solution is obtained only in a small neighborhood of $L$ (since $L$ is only assumed to be holomorphically convex). We shall indicate the necessary modifications to get a globally defined solution when $L$ is polynomially convex, with estimates near $L$. We need the following lemma which should be compared with Theorem 3.1 in [11]. We denote the variables on $C^{n+1}$ by $z$.

Lemma 4. (Notation as above) There exists a continuous plurisubharmonic exhausting function $\rho \geqq 0$ on $C^{n+1}$ such that

(a) $\rho^{-1}(0)=L=L_{0} \cup S$,

(b) $\rho(z) \leqq d(z, L)^{2}$ for $z$ near $L$, and

(c) $\rho(z)=d(z, S)^{2}$ for $z$ near $S \backslash U_{0}$.

We postpone the proof of Lemma 4 for a moment and continue with the proof of Lemma 1. We adopt the notation of [11]. For $\varepsilon>0$ set

$$
\omega_{\varepsilon}=\left\{z \in C^{n+1}: \rho(z)<\varepsilon^{2}\right\} .
$$

Choose $\varepsilon_{0}>0$ such that $\omega_{\varepsilon_{0}} \subset L_{\varepsilon_{0}} \cup U_{0}$ and Lemma 4 holds for $z \in \omega_{\varepsilon_{0}}$. For $0<\varepsilon \leqq \varepsilon_{0}$ we then have

$$
L(\varepsilon) \subset \omega_{\varepsilon} \subset L(\varepsilon) \cup U_{0}, \quad \omega_{\varepsilon} \backslash U_{0}=L(\varepsilon) \backslash U_{0}=S(\varepsilon) \backslash U_{0} .
$$

Recall that $f=\bar{\partial} X$ satisfies the estimate,

$$
|f(z)|=o\left(d(z, L)^{\gamma-1}\right)
$$

and $\left.f\right|_{U_{0}}=0$. Let $v=\operatorname{dim} S(=2$ in our case $)$. We get an estimate

$$
\int_{\omega_{3 s}}|f|^{2} d V=o\left(\varepsilon^{2(r-1)}\right) O\left(\varepsilon^{2 n}\right)=o\left(\varepsilon^{2(r+n-1)}\right)
$$


as $\varepsilon \rightarrow 0$, the extra term $O\left(\varepsilon^{2 n}\right)$ coming from the volume of the tube $S(3 \varepsilon)$. Fix an $\varepsilon, 0<\varepsilon \leqq \varepsilon_{0} / 3$. Let $\phi_{\varepsilon}=h_{\varepsilon} \circ \rho$, where $h_{\varepsilon}: \boldsymbol{R} \rightarrow \boldsymbol{R}_{+}$is a convex increasing function such that $h_{\varepsilon}(t)=0$ for $t \leqq 2 \varepsilon$, and $h_{\varepsilon}$ is increasing so fast on $t>2 \varepsilon$ that $\int_{C^{n+1} \backslash \omega_{3 \varepsilon}}|f|^{2} e^{-\phi_{\varepsilon}} d V$ is no larger than the integral in (6). Thus

$$
\int_{C^{n+1}}|f|^{2} e^{-\phi_{F}} d V=o\left(\varepsilon^{2(r+n-1)}\right) .
$$

According to Theorem 4.4.2 in [12] there is a solution of the equation $\bar{\partial} w_{\varepsilon}=f$ defined on all of $C^{n+1}$ such that

$$
\int_{C^{n+1}}\left|w_{\varepsilon}\right|^{2} e^{-\phi_{k}} \frac{d V}{\left(1+|z|^{2}\right)^{2}}=o\left(\varepsilon^{2(r+n-1)}\right) .
$$

Since $\phi_{\varepsilon}=0$ on the set $\omega_{2 \varepsilon} \subset \subset C^{n+1}$, we get

$$
\left\|w_{\varepsilon}\right\|_{L^{2}\left(\omega_{2 \varepsilon}\right)}=o\left(\varepsilon^{r+n-1}\right) .
$$

Since $L(2 \varepsilon) \subset \omega_{2 \varepsilon}$, the Cauchy estimates (see Lemma 4.4 in [11]) allow us to pass from the $L^{2}$ estimate on $\omega_{2 \varepsilon}$ to a sup norm estimate on $L(\varepsilon)$ :

$$
\left\|w_{\varepsilon}\right\|_{L^{\infty}(L(\varepsilon))}=O\left(\varepsilon^{-(n+1)}\right) o\left(\varepsilon^{r+n-1}\right)=o\left(\varepsilon^{r-2}\right) .
$$

The mapping $Y_{\varepsilon}=X-w_{\varepsilon}: C^{n+1} \rightarrow C^{n}$ is holomorphic on all of $C^{n+1}$ and it satisfies Lemma 1 for sufficiently small $\varepsilon>0$, provided that $r \geqq 3$. This proves Lemma 1, granted that Lemma 4 holds.

Proof of Lemma 4. Since $L$ is polynomially convex, there exists a smooth plurisubharmonic exhaustion function $\rho_{1} \geqq 0$ on $C^{n+1}$ such that $\rho_{1}^{-1}(0)=L$ and $\rho_{1}$ is strongly plurisubharmonic outside $L$ [12]. Since $\rho_{1}$ vanishes to second order on $L$, we may assume (replacing $\rho_{1}$ by $c \rho_{1}$ for a small $c>0$ if necessary) that $\rho_{1}(z) \leqq d(z, L)^{2}$. Thus (a) and (b) hold for $\rho_{1}$.

We now modify $\rho_{1}$ near $S \backslash U_{0}$ in order to satisfy (c) there. The function $\rho_{2}(z)=d(z, S)^{2}$ is strongly plurisubharmonic on a sufficiently small tubular neighborhood $V \subset C^{n+1}$ of $S \backslash U_{0}$. Choose a smooth real function $\chi$, with compact support contained in $U_{0} \cap V$, and such that $\left.\chi\right|_{b V \cap S}>0$. If $\delta>0$ is sufficiently small, the function $\rho_{2}^{\prime}=\rho_{2}-\delta \chi$ is still strongly plurisubharmonic in $V$, and $\rho_{2}^{\prime}=\rho_{2}$ near $S \backslash U_{0}$. Near the set $S \cap b V \subset \subset U_{0}$ we have $\rho_{2}^{\prime}<0 \leqq \rho_{1}$. Hence the function

$$
\rho_{3}=\max \left(\rho_{1}, \rho_{2}^{\prime}\right)
$$

is well defined, continuous and plurisubharmonic in a smaller neighborhood $W \subset U_{0} \cup V$ of $L$. We have $\rho_{3}=\rho_{1}$ near $L_{0}, \rho_{3}=\rho_{2}$ in $W \backslash U_{0}$, and $\rho_{3}^{-1}(0)=L$.

It remains to extend $\rho_{3}$ to an exhausting plurisubharmonic function $\rho$ on $C^{n+1}$ such that $\rho=\rho_{3}$ near $L$. This can be achieved by taking $\rho=$ $\max \left\{\rho_{3}, C\left(\rho_{1}-\eta\right)\right\}$ for a suitably large constant $C>0$ and a small constant $\eta>0$. This proves Lemma 4 . 
Proof of Lemma 2. Fix $x \in K$ and set $f(t)=\left|\phi_{t}(x)-\psi_{t}(x)\right|$. This function is defined for $0 \leqq t \leqq t_{0}$ for some $t_{0}>0$. We have

$$
\begin{aligned}
f(t) & =\left|\int_{0}^{t}\left(X_{s}\left(\phi_{s}(x)\right)-Y_{s}\left(\psi_{s}(x)\right)\right) d s\right| \\
& \leqq\left|\int_{0}^{t}\left(X_{s}\left(\phi_{s}(x)\right)-X_{s}\left(\psi_{s}(x)\right)\right) d s\right|+\left|\int_{0}^{t}\left(X_{s}\left(\psi_{s}(x)\right)-Y_{s}\left(\psi_{s}(x)\right)\right) d s\right| \\
& \leqq B \int_{0}^{t} f(s) d s+A(\varepsilon) .
\end{aligned}
$$

The Gronwall's inequality $[1$, p. 63] implies

$$
f(t) \leqq A(\varepsilon) e^{B t}
$$

for all $0 \leqq t \leqq 1$ where the flow $\psi_{t}(x)$ is defined. Since $A(\varepsilon) e^{B} \leqq \varepsilon$ by hypothesis, the above inequality shows that $\psi_{t}(x) \in K_{t}(\varepsilon)$ where it is defined. Hence $\psi_{t}(x)$ is defined for all $x \in K$ and all $0 \leqq t \leqq 1$. This proves Lemma 2.

\section{References}

1. Abraham, R., Marsden, J.E.: Foundations of Mechanics, 2nd. ed. Reading: Benjamin 1987

2. Alexander, H.: The polynomial hull of a rectifiable curve in $C^{n}$. Amer. J. Math. 110, 629-640 (1988)

3. Andersén, E.: Volume-preserving automorphisms of $C^{n}$. Complex Variables 14, 223-235 (1990)

4. Andersén, E., Lempert, L.: On the group of holomorphic automorphisms of $C^{n}$. Inventiones Math. 110, 371-388 (1992)

5. Forstneric, F.: Approximation by automorphisms on smooth submanifolds of $C^{n}$. Math. Ann. 300, 719-738 (1994)

6. Forstneric, F., Globevnik, J.: Discs in pseudoconvex domains. Comment. Math. Helvetici 67, 129-145 (1992)

7. Forstneric, F., Globevnik, J., Rosay, J.-P.: Non straightenable complex lines in $\boldsymbol{C}^{2}$. Preprint, April 1995

8. Forstneric, F., Rosay, J.-P.: Approximation of biholomorphic mappings by automorphisms of $C^{n}$. Invent. Math. 112, 323-349 (1993). Correction, Invent. Math. 118, 573-574 (1994)

9. Globevnik, J.: Relative embeddings of discs into convex domains. Invent. Math. 98, 331$350(1989)$

10. Globevnik, J., Stensønes, B.: Holomorphic embeddings of planar domains into $C^{2}$. Math. Ann. (1995)

11. Hörmander, L., Wermer, J.: Uniform approximation on compact sets in $\boldsymbol{C}^{\boldsymbol{n}}$. Math. Scand. 23, 5-21 (1968)

12. Hörmander, L.: An Introduction to Complex Analysis in Several Variables, 3rd ed. Amsterdam: North Holland 1990

13. Range, R.M. and Siu, Y.T.: $\mathcal{C}^{k}$ approximation by holomorphic functions and $\bar{\partial}$-closed forms on $\mathcal{C}^{k}$ submanifolds of a complex manifold. Math. Ann. 210, 105-122 (1974)

14. Rosay, J.-P.: Straightening of arcs. (Colloque d'analyse complexe et géométrie, Marseille, 1992, pp. 217-226.) Astérisque 217, 1993

15. Rosay, J.-P., Rudin, W.: Holomorphic maps from $C^{n}$ to $C^{n}$. Trans. Amer. Math. Soc. $310,47-86(1988)$ 
16. Rosay, J.-P., Rudin, W.: Holomorphic embeddings of $C$ in $C^{n}$. In Several complex variables: Proceedings of the Mittag Leffler Institute, 1987-88, J.E. Fornaess Ed., 563569, Math. Notes 38, Princeton Univ. Press (1993)

17. Stolzenberg, G.: Polynomially and rationally convex sets. Acta Math. 109, 259-289 (1963) 